\title{
Pre-pregnancy predictors of hypertension in pregnancy among Aboriginal and Torres Strait Islander women in north Queensland, Australia; a prospective cohort study
}

Sandra K Campbell ${ }^{1 *}$, John Lynch², Adrian Esterman ${ }^{3}$ and Robyn McDermott ${ }^{4}$

\begin{abstract}
Background: Compared to other Australian women, Indigenous women are frequently at greater risk for hypertensive disorders of pregnancy. We examined pre-pregnancy factors that may predict hypertension in pregnancy in a cohort of Aboriginal and Torres Strait Islander women in north Queensland.

Methods: Data on a cohort of 1009 Indigenous women of childbearing age (15-44 years) who participated in a 1998-2000 health screening program in north Queensland were combined with 1998-2008 Queensland hospitalisations data using probabilistic data linkage. Data on the women in the cohort who were hospitalised for birth $(n=220)$ were further combined with Queensland perinatal data which identified those diagnosed with hypertension in pregnancy.

Results: Of 220 women who gave birth, 22 had hypertension in the pregnancy after their health check. The mean age of women with and without hypertension was similar (23.7 years and 23.9 years respectively) however Aboriginal women were more affected compared to Torres Strait Islanders. Pre-pregnancy adiposity and elevated blood pressure at the health screening program were predictors of a pregnancy affected by hypertension. After adjusting for age and ethnicity, each $1 \mathrm{~cm}$ increase in waist circumference showed a 4\% increased risk for hypertension in pregnancy (PR 1.04; 95\% Cl; 1.02-1.06); each 1 point increase in BMI showed a 9\% adjusted increase in risk (1.09; 1.04-1.14). For each $1 \mathrm{mmHg}$ increase in baseline systolic blood pressure there was an age and ethnicity adjusted $6 \%$ increase in risk and each $1 \mathrm{mmHg}$ increase in diastolic blood pressure showed a $7 \%$ increase in risk (1.06; 1.03-1.09 and 1.07; 1.03-1.11 respectively). Among those free of diabetes at baseline, the presence of the metabolic syndrome (International Diabetes Federation criteria) predicted over a three-fold increase in age-ethnicity-adjusted risk (3.5; 1.50-8.17).
\end{abstract}

Conclusions: Pre-pregnancy adiposity and features of the metabolic syndrome among these young Aboriginal and Torres Strait Islander women track strongly to increased risk of hypertension in pregnancy with associated risks to the health of babies.

Keywords: Indigenous, Pre-pregnancy, Hypertension, Preeclampsia

\footnotetext{
* Correspondence: Sandra.Campbell@postgrads.unisa.edu.au

${ }^{1}$ School of Health Sciences, Division of Health Sciences, University of South Australia, City East Campus North Terrace (P5-21), GPO Box 2471, Adelaide, SA 5001, Australia

Full list of author information is available at the end of the article
} 


\section{Background}

Hypertensive disorders complicate $5-10 \%$ of pregnancies and contribute greatly to maternal and fetal morbidity and mortality throughout the world [1-3]. Populationbased Australian studies undertaken in Victoria [4] and South Australia [5] have shown a prevalence of 9.8\% and $9.2 \%$ respectively, however, Australian Indigenous women have been reported as having 66\% greater risk for pregnancy-induced hypertension when compared with non-Indigenous women [6]. Australian Aboriginal and Torres Strait Islander women are also at comparatively higher risk for developing chronic hypertension and cardiovascular disease at a younger age than other Australian women $[7,8]$. Poor cardiovascular health prior to pregnancy modulates maternal preeclamptic syndrome so that frequency and severity of hypertensive disease in pregnancy are increased [9]. Women with chronic hypertension may have uneventful pregnancies when their hypertension remains mild to moderate, however, overall outcomes are poorer than for normotensive women and they are at at greater risk of developing preeclampsia $(\geq 20 \%)$ [10]. Preeclampsia occurs in about $3-5 \%$ of pregnancies [11] and is the hypertensive condition whether it occurs alone or superimposed on chronic hypertension most often associated with maternal and fetal complications including fatalities [1]. The disorder is an early marker not only for future maternal cardiovascular and metabolic disease, $[1,11,12]$ but also confers long-term risk for cardiovascular disease among infants [2]. Hypertension during pregnancy has been identified as a predictor of poor birth outcomes in Aboriginal and Torres Strait Islander women in north Queensland [13]. However, it remains unclear why this group of women have an increased risk for hypertension in pregnancy.

Pre-pregnancy overweight and obesity have been identified as risk factors for hypertensive disorders of pregnancy $[14,15]$. In the United States, obesity is the leading risk factor for preeclampsia; it confers a threefold increased risk and is present in 30\% of cases [16]. Now a major epidemic in developed countries including Australia, obesity is impacting on the health and wellbeing of Indigenous Australians. With the change from traditional carbohydrate based diets to energy dense high fat diets, combined with lower levels of physical activity [17], rates of obesity among Aboriginal and Torres Strait Islander women of childbearing age have escalated [18]. We examined pre-pregnancy factors that may predict any hypertension in pregnancy in a cohort of Aboriginal and Torres Strait Islander women in north Queensland. We hypothesised that abnormal prepregnancy blood pressure levels and maternal adiposity would increase the risk of hypertensive disorders during pregnancy.

\section{Methods}

\section{Study population}

The Aboriginal and Torres Strait Islander women in this study were participants in the March 1998-December 2000 Well Person's Health Check (WPHC) crosssectional health survey [19], which included 26 rural and remote Indigenous communities in the Bowen, Cairns, Cape York, Torres Strait and Mount Isa Health service districts. All adults were invited to participate through printed media, local radio and word of mouth via the local health service, community council and community groups. The survey was attended by 2862 Indigenous people aged 15 years and over, giving an overall participation rate of $44.5 \%$ (according to local census data) [20]; 51.7\% were female. Participants overall, were not different demographically from the age and sex distribution of the Indigenous population who did not attend, based on census data. A subset of women from the original survey data were selected for this study because the WPHC occurred when they were aged between 15-44 years (a generally accepted range of childbearing age).

\section{Data sources}

Three datasets were linked to bring together information on maternal pre-pregnancy factors (anthropometric, biochemical and lifestyle) gathered at the WPHC, and the presence or absence of a hypertensive disorder during the next pregnancy following this health check. First, records of Aboriginal and Torres Strait Islander women of childbearing age who attended the WPHC (details of the survey are outlined below) were linked to Queensland hospital Unit Registration numbers by probabilistic matching of name, date of birth and residential address. The second dataset contained hospitalisations data (including International Classification of Disease (ICD) 9 and ICD 10 codes and adjacent diagnosis-related groups and descriptions) identified those women who were hospitalised for pregnancy-related conditions after the date of their WPHC up to the study censor date on 30 March 2008. To idenify women who had a hypertensive disorder of pregnancy after the WPHC, a third dataset provided by Statistical Output, Health Statistics Centre, Queensland Health was added. The third dataset included perinatal data specific to those women who had been hospitalised for birth of an infant of at least 20 weeks completed gestation or a birthweight $>400 \mathrm{~g}$ when gestation was unknown [21]. When a woman was hospitalised for more than one birth during the followup period, only perinatal data for the birth nearest the health check was requested. Women who were likely to have been pregnant at the time of their health check were excluded based on the date of their health check, the date of their delivery and the estimated gestation of the pregnancy at the time of birth. 
ICD codes within the perinatal data identified women who had hypertensive conditions (ICD O10-O16) during their pregnancy (Figure 1). The outcome measure "hypertension in pregnancy" in this study applies to women who were recorded in the perinatal dataset as receiving a diagnosis of one of the forms of the disorder; pre-existing hypertension (ICD O10), pre-existing hypertension with superimposed preeclampsia-eclampsia (ICD O11), gestational hypertension without proteinuria (ICD 013) and preeclampsia-eclampsia (ICD O14 and O15).

\section{Well person's health check}

A standardised proforma was used for data collection in the baseline WPHC survey. Body measurements and specimen collection were performed by a multidisciplinary team of trained staff from the Cairns Tropical Public Health Unit (Queensland Health) and local health staff in each communtiy. Face-to-face interviews followed a structured questionnaire. Fasting venous blood samples were collected by a medical officer, registered nurse or trained phlebotomist [19].

\section{Anthropometric measures}

Participants were invited to attend in the early morning following at least an $8 \mathrm{~h}$ fast. They were weighed in light clothing to the nearest $0.1 \mathrm{~kg}$ on digital electronic scales and height was measured to the nearest centimetre. Body mass index (BMI) was calculated as weight $(\mathrm{kg})$ divided by height squared $\left(\mathrm{m}^{2}\right)$. Waist circumference was measured to the nearest centimetre. Anthropometric cut-offs were set according to World Health Organisation criteria [22].

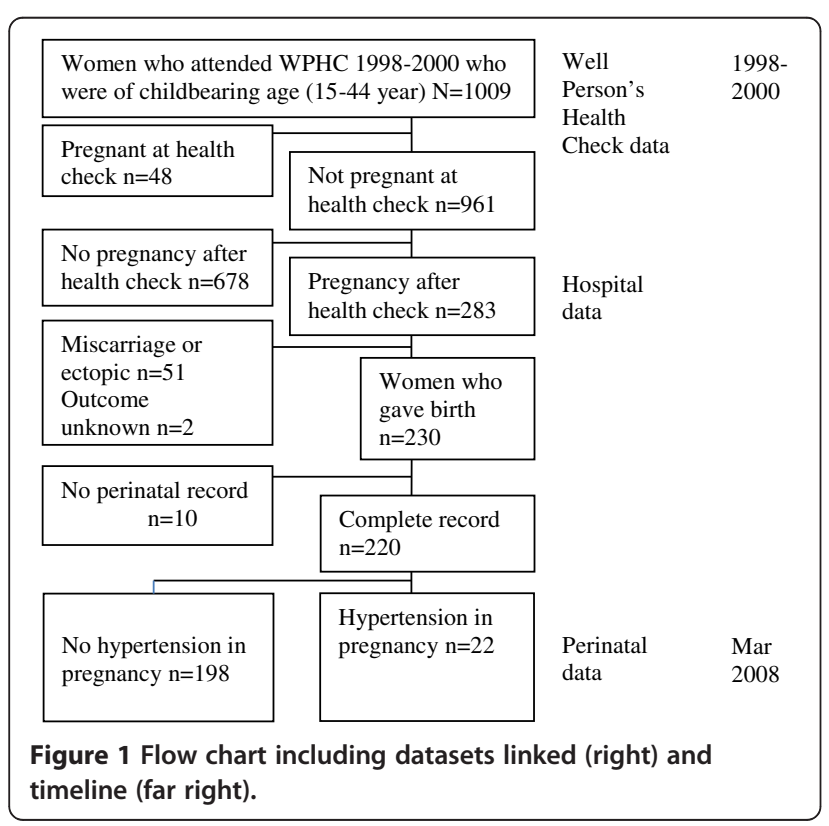

\section{Blood pressure}

Seated blood pressure was recorded three times over approximately $10 \mathrm{~min}$ with an automated blood pressure monitor, and mean systolic and diastolic measurements were calculated.

\section{Self-reported behavioural factors}

Fruit and vegetable intake was self-reported by 24-h recall and 7-day recall was used to assess the duration and intensity of physical activity. The participants were categorised as having adequate physical activity if they reported a minimum of $30 \mathrm{~min}$ of moderate to vigorous exercise on at least 5 days in the week. Current smoking status was self-reported. Participants who drank alcohol self-reported the types and amounts of alcohol consumed in the week before the health check; they were then categorised as being safe drinkers, non-drinkers or harmful ("risky") drinkers according to Australian National Health and Medical Research Council guidelines (2001). Risky drinking for women was defined as more than two standard drinks per day or more than four on any single occasion. The physical activity, smoking and alcohol intake measures have been widely used [23] and the 24-h recall dietary questionnaire and red cell folate measure have been validated against other measures of micronutrient intake and the quality of the diet with respect to fruit and vegetables [24,25].

\section{Biochemical measures}

Early-morning fasting venous blood samples were collected in ethylenediamine tetra-acetic acid vacuum tubes and clotted serum separator vacuum tubes (spun for $10 \mathrm{~min}$ at $1000 \mathrm{RCF}$ in a swinging bucket centrifuge and separated within $1 \mathrm{~h}$ ). Samples were stored in $4-8^{\circ} \mathrm{C}$ and transported for analysis within 24 hours. The biochemical measurements included glucose, triglycerides, total cholesterol, high density lipoprotein cholesterol, $\gamma$-glutamyl transferase and red cell folate. Glucose and lipids were measured using photometric enzyme endpoint assay with Cobas Integra 700/400 (Roche Diagnostic, Switzerland). $\gamma$-Glutamyl transferase was measured by a kinetic photometric procedure with Cobas Integra 800 (Roche Diagnostic, Switzerland). An elevated level was defined as $\geq 50 \mathrm{U} / \mathrm{L}$ and $<50 \mathrm{U} / \mathrm{L}$ as normal, according to criteria established by Queensland Health Pathology Service (http://www.health. qld.gov.au/qhcss/qhps/default.asp). Red cell folate was measured with the Bayer Advia Centaur automated immunoassay system (Bayer, Australia) by the Queensland Health Pathology Service in Brisbane. The reference range for this assay was 295-1800 $\mathrm{nmol} / \mathrm{L}$.

Urine specimens collected in sterile $50-\mathrm{ml}$ containers were either the first morning or at least $2 \mathrm{~h}$ after the most recent void. If protein was detected by dipstick urinalysis (Combur-test, Roche) or if the participant was 
known to have diabetes, hypertension or obesity (BMI $>30 \mathrm{~kg} / \mathrm{m}^{2}$ ), the albumin: creatinine ratio was measured by immunoassay in grams per mole. Microalbuminuria was defined as an albumin:creatinine ratio of 3.4-34 g/mol, and macroalbuminuria as $>34 \mathrm{~g} / \mathrm{mol}$ [26]. From May 1999 to the end of the project, the albumin:creatinine ratio was measured in all urine specimens.

\section{Metabolic syndrome}

The metabolic syndrome in this study is defined according to the International Diabetes Federation criteria as comprising waist circumference $\geq 80 \mathrm{~cm}$ plus two or more of the following: raised triglycerides $(\geq 1.7 \mathrm{mmol} / \mathrm{L})$, reduced high-density lipoprotein $(<1.29 \mathrm{mmol} / \mathrm{L})$, raised blood pressure (systolic $\geq 130 \mathrm{~mm} \mathrm{Hg}$ or diastolic $\geq 85 \mathrm{~mm} \mathrm{Hg}$ ) and raised plasma glucose $(\geq 5.6 \mathrm{mmol} / \mathrm{L})$ (http://www.idf. org/metabolic-syndrome).

\section{Ethics}

Ethics approval for the WPHC was provided by the Cairns Base Hospital Ethics Committee in March 1998. The participants provided consent to have their Queensland Health medical records reviewed and linked to their WPHC records. The current data linkage study was approved by the University of South Australia and Cairns Base Hospital Human Research Ethics Committees (June 2009 and September 2009) with support from the peak health councils representing Indigenous people in the region: Apunipima and the Torres Strait Islander and Northern Peninsula Area Health Councils.

\section{Statistical analysis}

The data were analysed in a generalised linear model (Poisson distribution) with robust variance estimates to calculate prevalence ratios (PRs) and 95\% confidence intervals (CIs) with 2 sided p-values of 0.05 for baseline characteristics associated with hypertension during the first pregnancy after their health check. Data are presented in both unadjusted and adjusted analyses. An a priori decision was taken to adjust the model for age because hypertension is commonly associated with increasing age and, because it was expected that Aboriginal and Torres Strait Islander women would differ in their anthropometic and metabolic characteristics, ethnicity was included in the adjusted model. Further adjustment for months from participation in the baseline survey to time of admission for birth was not associated with the outcome and was not retained. Fruit and vegetable intake was excluded from the analyses due to very small cell sizes. The analysis was conducted with STATA 11 (STATAcorp, College Station, Texas, USA).

\section{Results}

Pre-pregnancy characteristics at the baseline health check At the baseline WHPC, there were 1009 women of childbearing age (15-44 years) in the study sample. We excluded 48 women because they were pregnant at the time of their health check. Of the remaining 961 women, $61.2 \%$ identified as Aboriginal, 30.7\% as Torres Strait Islander, and $8.1 \%$ identified as both Torres Strait Islander and Aboriginal. Characteristics of the cohort (demographics, waist circumferences BMIs, smoking status, alcohol use, fruit and vegetable intake, physical activity, blood pressure, blood lipids, red cell folate, fasting glucose, kidney and liver function tests, prevalences of metabolic syndrome and diabetes) are reported in detail elsewhere [20]. Abdominal obesity (waist circumference $\geq 88 \mathrm{~cm}$ ) was found in $60.5 \%$ of women, and $37.1 \%$ had a BMI $>30$. Adequate fruit and vegetable intake and physical activity were low (1.5 and 21\% respectively), and self-reported rates of tobacco smoking and risky drinking in the week before the baseline health check were high $(60.8 \%$ and $41.9 \%$, respectively).

\section{Prevalence of hypertension in pregnancy}

Figure 1 shows that 283 women were hospitalised for a pregnancy-related condition in the time between their WPHC in 1998-2000 and March 2008; 51 pregnancies ended in miscarriage or because of an ectopic pregnancy, and two pregnancy outcomes were unknown. In the time up to the censor date, 230 women were hospitalised for birth. Ten of these women were excluded because perinatal records were not matched. Of the remaining 220 women (21.4\% were primiparous), 9 had pre-existing hypertension at the time of their WPHC. Seven women with normal blood pressure at baseline developed gestational hypertension (new-onset hypertension after mid-pregnancy, in the absence of proteinuria). Seven women (3.2\%) developed preeclampsia (hypertension and proteinuria); six cases were in women with normal baseline blood pressure and were described as moderate, and one severe case was superimposed on pre-existing hypertension. Overall, 22 women (10\%) had a hypertensive disorder in their first pregnancy following the baseline WPHC.

\section{Pre-pregnancy characteristics as risk for hypertension in pregnancy: unadjusted analyses}

Unadjusted analysis in Table 1 shows that of the 22 women who had hypertension in the first pregnancy after their health check, $72.7 \%$ were Aboriginal; the remaining $27.3 \%$ of the women identified as Torres Strait Islander or, both Torres Strait Islander and Aboriginal ( $p=0.157)$. There was no difference in mean age between the women who did and did not have hypertension in pregnancy $(\mathrm{p}=0.914)$. Elevated pre-pregnancy 
Table 1 Baseline characteristics of the cohort, by hypertension in pregnancy $(n=220)$

\begin{tabular}{|c|c|c|c|c|c|c|}
\hline \multirow[t]{2}{*}{ Pre-pregnancy characteristic } & \multicolumn{2}{|c|}{$\begin{array}{l}\text { No hypertension during pregnancy } \\
\qquad(\mathrm{n}=198)\end{array}$} & \multicolumn{2}{|c|}{$\begin{array}{l}\text { Hypertension during pregnancy } \\
\qquad(n=22)\end{array}$} & \multirow[b]{2}{*}{ Unadjusted PR (95\% Cl) } & \multirow[b]{2}{*}{$p$ value } \\
\hline & $\mathrm{n}$ & $\%$ of $n$ or mean $(s d)$ & $\mathrm{n}$ & $\%$ of $n$ or mean $(\mathbf{s d})$ & & \\
\hline \multicolumn{7}{|l|}{ Indigenous group } \\
\hline Aboriginal & 112 & 56.6 & 16 & 72.7 & 1.00 & \\
\hline Torres Strait Islander ${ }^{\mathrm{a}}$ & 86 & 43.4 & 6 & 27.3 & $0.52(0.21-1.28)$ & 0.157 \\
\hline Age years (mean, sd) & 198 & $23.9(5.4)$ & 22 & $23.7(6)$ & $0.99(0.92-1.07)$ & 0.914 \\
\hline \multicolumn{7}{|l|}{ Waist circumference (cm) } \\
\hline$<80$ & 65 & 33 & 5 & 22.7 & 1.00 & \\
\hline $80-87.9$ & 40 & 20.3 & 2 & 9.1 & $0.67(0.13-3.30)$ & 0.619 \\
\hline$\geq 88$ & 92 & 46.7 & 15 & 68.2 & $1.96(0.74-5.17)$ & 0.372 \\
\hline Waist circumference (mean, sd) & 197 & $88.7(15.8)$ & 22 & $95.4(17.6)$ & $1.02(1.00-1.04)$ & 0.054 \\
\hline \multicolumn{7}{|l|}{ BMI category $\left(\mathrm{kg} / \mathrm{m}^{2}\right)$} \\
\hline$<18.5$ & 23 & 11.6 & 0 & 0 & & \\
\hline $18.5-25$ & 74 & 37.4 & 7 & 31.8 & 1.00 & \\
\hline 25-30 & 48 & 24.2 & 6 & 27.3 & $1.29(0.46-3.63)$ & 0.635 \\
\hline$\geq 30+$ & 53 & 26.7 & 9 & 40.9 & $1.68(0.66-4.26)$ & 0.276 \\
\hline BMI (mean, sd) & 198 & $26.1(7)$ & 22 & $29(6.5)$ & $1.05(1.00-1.09)$ & 0.033 \\
\hline \multicolumn{7}{|l|}{ Smoker } \\
\hline No & 57 & 28.9 & 8 & 36.4 & 1.00 & \\
\hline Yes & 140 & 71.1 & 14 & 63.6 & $0.74(0.33-3.68)$ & 0.469 \\
\hline \multicolumn{7}{|l|}{ Alcohol use } \\
\hline Safe drinker & 56 & 28.9 & 4 & 18.2 & 1.00 & \\
\hline Non drinker & 64 & 33 & 7 & 31.8 & $1.48(0.45-4.82)$ & 0.517 \\
\hline Risky drinker & 74 & 38.1 & 11 & 50 & $1.94(0.65-5.82)$ & 0.236 \\
\hline \multicolumn{7}{|l|}{ Enough physical activity } \\
\hline No & 160 & 80.8 & 17 & 77.3 & 1.00 & \\
\hline Yes & 38 & 19.2 & 5 & 22.7 & $1.23(0.47-3.11)$ & 0.691 \\
\hline \multicolumn{7}{|l|}{ Systolic blood pressure $(\mathrm{mmHg})$} \\
\hline$\leq 120$ & 142 & 72.1 & 8 & 36.4 & 1.00 & \\
\hline$>120$ & 55 & 27.9 & 14 & 63.6 & $3.80(1.67-8.67)$ & 0.001 \\
\hline \multicolumn{7}{|l|}{ Diastolic blood pressure $(\mathrm{mmHg})$} \\
\hline$\leq 80$ & 190 & 96.5 & 19 & 86.4 & 1.00 & \\
\hline$>80$ & 7 & 3.5 & 3 & 13.6 & $3.30(1.16-9.35)$ & 0.025 \\
\hline \multicolumn{7}{|l|}{ Cholesterol (mg/dL) } \\
\hline$\leq 5.5$ & 176 & 90.3 & 20 & 90.9 & 1.00 & \\
\hline > 5.5 (elevated) & 19 & 9.7 & 2 & 9.1 & $0.93(0.23-3.73)$ & 0.922 \\
\hline \multicolumn{7}{|l|}{ Triglycerides (mmol/L) } \\
\hline$\leq 2$ & 173 & 89.6 & 19 & 86.4 & 1.00 & \\
\hline$>2$ (elevated) & 20 & 10.4 & 3 & 13.6 & $1.31(0.42-4.12)$ & 0.635 \\
\hline \multicolumn{7}{|l|}{ HDL cholesterol (mmol/L) } \\
\hline$>1$ & 108 & 56.5 & 9 & 40.9 & 1.00 & \\
\hline$\leq 1$ (low) & 83 & 43.5 & 13 & 59.1 & $1.76(0.78-3.95)$ & 0.170 \\
\hline
\end{tabular}


Table 1 Baseline characteristics of the cohort, by hypertension in pregnancy $(\mathbf{n}=\mathbf{2 2 0})$ (Continued)

\begin{tabular}{|c|c|c|c|c|c|c|}
\hline \multicolumn{7}{|c|}{ Red cell folate (nmol/L) } \\
\hline$\geq 295$ & 126 & 65 & 17 & 77.3 & 1.00 & \\
\hline$<295$ (low) & 68 & 35 & 5 & 22.7 & $0.58(0.22-1.50)$ & 0.260 \\
\hline \multicolumn{7}{|c|}{ Fasting glucose (mmol/L) } \\
\hline$\leq 5.5$ & 182 & 93.3 & 20 & 90.9 & 1.00 & \\
\hline$>5.5$ & 13 & 6.7 & 2 & 9.1 & $1.35(0.35-5.24)$ & 0.668 \\
\hline \multicolumn{7}{|c|}{ Albumin:creatinine ratio (mg/mmol) } \\
\hline$\leq 3.4$ & 121 & 82.3 & 13 & 68.4 & 1.00 & \\
\hline$>3.4$ & 26 & 17.7 & 6 & 31.6 & $2.25(0.39-13.05)$ & 0.366 \\
\hline \multicolumn{7}{|c|}{ Gamma-glutamyl transferase (U/L) } \\
\hline$\leq 50$ & 183 & 93.9 & 20 & 90.9 & 1.00 & \\
\hline$>50$ (elevated) & 12 & 6.1 & 2 & 9.1 & $1.45(0.37-5.60)$ & 0.590 \\
\hline \multicolumn{7}{|c|}{ Metabolic syndrome ${ }^{b}$} \\
\hline No & 153 & 80.1 & 12 & 57.1 & 1.00 & \\
\hline Yes & 38 & 19.9 & 9 & 42.9 & $2.63(1.18-5.88)$ & 0.018 \\
\hline
\end{tabular}

systolic and diastolic blood pressure at the WPHC predicted more than a three-fold risk for hypertension in pregnancy $(\mathrm{p}=0.001$ and 0.025 respectively). The affected women were more likely to have a higher mean waist circumference $(\mathrm{p}=0.054)$ and a mean BMI three points above the women with no hypertension $(p=0.033)$. More women who had hypertension reported risky drinking (50\% versus $38 \%$; $\mathrm{p}=0.236$ ) and elevated albumin:creatinine ratio and low levels of high density lipoprotein cholesterol were more common (31.6\% versus $17.7 \%$ and $59.1 \%$ versus $43.5 \%$ respectively) though not statistically significant ( $\mathrm{p}=0.366$ and 0.170 respectively). Non-diabetic women who were diagnosed with hypertension in their pregnancy had more than twice the prevalence of features of the metabolic syndrome at the baseline health check ( $42.9 \%$ versus $19.9 \% ; \mathrm{p}=0.018$ ). Among all the women who had a baby $(n=220)$, baseline self-reported levels of adequate physical activity were low (19.5\%) and smoking rates and risky drinking were high (70\% and $38.6 \%$ respectively).

\section{Pre-pregnancy characteristics as risk for hypertension in pregnancy: analysis adjusted for age and ethinicity}

In a multivariable analysis (Table 2), a strong predictor for having hypertension in pregnancy was elevated baseline blood pressure. For each $1 \mathrm{mmHg}$ increase in prepregnancy systolic blood pressure there was an age and ethnicity adjusted 6\% increase in risk of hypertension in pregnancy (PR 1.06; 1.03-1.09) and for each $1 \mathrm{mmHg}$ increase in diastolic blood pressure there was a $7 \%$ increase in risk (1.07; 95\% CI 1.03-1.11). Body fat was also an important risk factor and for each $1 \mathrm{~cm}$ increase in waist circumference there was a $4 \%$ age and ethnicity adjusted increase in risk of hypertension in pregnancy (1.04;1.021.06) and for each 1 point increase in BMI, there was a $9 \%$ increase in risk $(1.09$; 1.04-1.14). The presence of metabolic syndrome at baseline predicted over a three-fold increase in adjusted risk among women free of diabetes at baseline $(3.5 ; 1.50-8.17)$. For each $1 \mathrm{mg} / \mathrm{mmol}$ increase in pre-pregnancy albumin:creatine ratio there was an age and ethnicity adjusted $1 \%$ increased risk $(1.01 ; 1.00-1.02)$. Pre-pregnancy alcohol use was not significantly associated with hypertension in pregnancy however, for each $1 \mathrm{U} / \mathrm{L}$ increase in $\gamma$-glutamyl transferase, possibly related to nonalcoholic fatty liver disease, there was a $1 \%$ increase in adjusted risk (1.01: 1.00-1.02). Pre-pregnancy smoking, physical activity, fasting glucose, red cell folate and blood fats were not statistically significantly associated with hypertension in pregnancy in this study.

\section{Discussion}

Twenty-two of the 220 women who gave birth in the 8-10 years after their health check had hypertension during their pregnancy. Seven of these women (3.2\%) were diagnosed with moderate $(n=6)$ or severe $(n=1)$ preeclampsia, a rate equivalent to that found among other population groups [11]. The strongest pre-pregnancy predictors of hypertension during pregnancy in this cohort of Aboriginal and Torres Strait Islander women were central adiposity (assessed by waist circumference), obesity (assessed by BMI) and an elevated blood pressure 
Table 2 Multivariable analysis for effects of pre-pregnancy factors and hypertension during pregnancy $(\mathbf{n}=\mathbf{2 2 0})$

\begin{tabular}{|c|c|c|c|c|c|}
\hline $\begin{array}{l}\text { Pre-pregnancy } \\
\text { characteristic }\end{array}$ & Unit increase & $\begin{array}{l}\text { Crude PR } \\
\text { (95\% confidence interval) }\end{array}$ & $p$ value & $\begin{array}{l}\text { PR adjusted for age and ethnicity } \\
\text { (95\% confidence interval) }\end{array}$ & $p$ value \\
\hline \multicolumn{6}{|l|}{ Indigenous group } \\
\hline Aboriginal & - & 1.00 & & & \\
\hline Torres Strait Islander ${ }^{\mathrm{a}}$ & - & $0.52(0.21-1.28)$ & 0.16 & & \\
\hline Age & "1 year" & $1.00(0.92-1.07)$ & 0.91 & & \\
\hline Waist circumference & "1 cm" & $1.02(1.00-1.04)$ & 0.05 & $1.04(1.02-1.06)$ & 0.001 \\
\hline BMI (kg/m2) & "1 unit" & $1.05(1.00-1.09)$ & 0.03 & $1.09(1.04-1.14)$ & $<0.001$ \\
\hline \multicolumn{6}{|l|}{ Alcohol intake } \\
\hline Safe drinker & - & 1.00 & & 1.00 & \\
\hline Non drinker & - & $1.47(0.45-4.82)$ & 0.52 & $1.38(0.43-4.47)$ & 0.59 \\
\hline Risky drinker & - & $1.94(0.64-5.91)$ & 0.24 & $1.79(0.58-5.49)$ & 0.31 \\
\hline Non-smoker & Smoker & $0.74(0.33-1.68)$ & 0.47 & $0.68(0.30-1.55)$ & 0.36 \\
\hline Enough physical activity & $<30 \mathrm{~min} /$ day 5 days per week & $0.83(0.32-2.12)$ & 0.69 & $0.78(0.31-1.55)$ & 0.60 \\
\hline Systolic blood pressure & "1 mmHg" & $1.05(1.02-1.07)$ & $<0.001$ & $1.06(1.03-1.09)$ & $<0.001$ \\
\hline Diastolic blood pressure & "1 mmHg" & $1.06(1.03-1.10)$ & $<0.001$ & $1.07(1.03-1.11)$ & $<0.001$ \\
\hline Cholesterol & "1 mmol/L" & $1.27(0.88-1.83)$ & 0.21 & $1.38(0.93-2.06)$ & 0.10 \\
\hline Triglycerides & "1 mmol/L" & $1.18(0.96-1.46)$ & 0.12 & $1.17(0.92-1.49)$ & 0.20 \\
\hline HDL cholesterol ( $\leq 1$ = low) & "1 mmol/L" & $0.74(0.13-4.22)$ & 0.73 & $0.61(0.09-3.82)$ & 0.60 \\
\hline Red cell folate & "1 nmol/L" & $1.00(1.00-1.01)$ & 0.006 & $1.00(1.00-1.01)$ & 0.009 \\
\hline Fasting glucose & "1 mmol/L" & $1.06(0.82-1.37)$ & 0.67 & $1.14(0.87-1.50)$ & 0.33 \\
\hline Albumin:creatinine ratio & "1 mg/mmol" & $1.01(1.00-1.02)$ & 0.02 & $1.01(1.00-1.02)$ & 0.003 \\
\hline Gamma-glutamyl transferase & "1 U/L" & $1.01(1.00-1.02)$ & 0.02 & $1.01(1.00-1.02)$ & 0.003 \\
\hline Hyper-triglyceridemic waist ${ }^{\mathrm{b}}$ - No & Yes & $1.55(0.40-5.93)$ & 0.53 & $1.53(0.39-6.01)$ & 0.54 \\
\hline Metabolic syndrome $e^{c}-$ No & Yes & $2.63(1.18-5.88)$ & 0.02 & $3.50(1.50-8.17)$ & 0.004 \\
\hline
\end{tabular}

Denominators vary because of missing values.

${ }^{a}$ Women in this group self-identified as Torres Strait Islander or, as both Torres Strait Islander and Aboriginal origin.

${ }^{b}$ Defined as raised triglycerides ( $>2 \mathrm{mmol} / \mathrm{l}$ and waist circumference $\geq 88 \mathrm{~cm}$ ).

'Defined by International Diabetes Federation criteria as comprising waist circumference.

$\geq 80 \mathrm{~cm}$ plus two or more of the following: raised triglycerides $(\geq 1.17 \mathrm{mmol} / \mathrm{l})$, reduced high-density lipoprotein $(<1.29 \mathrm{mmol} / \mathrm{L})$, raised blood pressure (systolic $\geq 130 \mathrm{mmHg}$ or diastolic $\geq 85 \mathrm{mmHg}$ ) and raised plasma glucose ( $\geq 5.6 \mathrm{mmol} / \mathrm{L}$ ). Women with diabetes excluded.

recording at the WPHC cross-sectional survey. By studying a sample of the Indigenous populations of Australia, which have some of the highest prevalence of hypertensive pregnancy, we have emphasised pre-pregnancy obesity and hypertension as independent contributors to this disease. Both of these risk factors are potentially amenable to intervention before pregnancy.

Our results add to previous Australian research that has identified elevated pre-pregnancy BMI as an important factor in the development of serious hypertensive disorders of pregnancy [27]. A systematic review of international controlled studies of risk factors for preeclampsia at antenatal booking showed that raised BMI before pregnancy (relative risk $2.47 ; 1.66-3.67$ ) is one of the few amenable to intervention [28]. Other factors known to increase risk that cannot be altered prior to pregnancy include previous history or family history of preeclampsia, multiple pregnancy, nulliparity, and maternal age among multiparous women. Our study highlights the need to focus health promotion efforts on encouraging healthy body weight among Aboriginal and Torres Strait Islander women of childbearing age.

Elevated pre-pregnancy blood pressure in this study was an additional predictor of a hypertensive disorder of pregnancy. Hypertension among Indigenous women and men aged $25-50$ years has previously been shown to be highly prevalent in communities included in the study [12]. Pre-pregnancy blood pressure assessment in this high risk population is important because undiagnosed chronic hypertension can be masked in early pregnancy due to physiological changes that may cause initial decreases in blood pressure, then misdiagnosed later in the pregnancy as a gestational disorder when abnormal readings re-surface [1]. One of the best predictors of chronic hypertension for Aboriginal and Torres Strait Islander Australians is overweight and obesity established by elevated waist circumference [17].

Aside from pre-pregnancy adiposity and raised blood pressure, two additional risk factors associated with poor 
metabolic health, elevated albumin:creatinine and the $\gamma$-glutamyl transferase, increased the risk for hypertension during pregnancy among the cohort. Raised albumin:creatine ratio as a marker of kidney damage and raised $\gamma$-glytamyl transferase as a marker of non-alcoholic fatty liver disease or risky alcohol use, provide a general picture of poor health in women of childbearing age. Among women in the cohort free of diabetes at baseline, presence of metabolic syndrome as defined by the International Diabetes Federation criteria, showed over a three-fold risk for hypertension during pregnancy. From a population health perspective, health risks among this cohort are exacerbated by high rates of smoking and risky alcohol use and low levels of physical activity. The extent of health challenges faced by these young women, and our improved understanding of how 'fetal programming' predicts the immediate and long term health of the next generation, call for interventions well before pregnancy begins in this population.

Encouraging weight loss during pregnancy is not a suitable strategy; weight loss outside of pregnancy requires lowering caloric intake or raising energy expended. Programs that incorporate a combination of physical activity and nutrition education have been shown to reduce body weight [29] and blood pressure [30,31]. In this study, less than $20 \%$ of the women reported undertaking adequate physical activity in the week before their baseline health check. It has been shown that when women exercise between 3 months and 1 year before or during pregnancy, the risk of preeclampsia is reduced [32,33]. The benefits of exercise prior to pregnancy for reducing the risk of preeclampsia, suggest that weight reduction interventions should feature well-designed physical activity components that focus on fitness, nutrition and weight loss in preference to weight loss alone.

This study is limited by its small sample size and low participation rate of eligible women $(<50 \%)$ in the baseline survey and there is a possible downward and upward response bias to self-reported variables including current smoking status, alcohol use, fruit and vegetable intake and levels of physical activity. Despite the low participation rate, the cohort was well characterised at baseline with standardised body and biochemical measurements and the internal validity of the associations found between baseline risk and later hypertension in pregnancy should be high. A potential limitation of the study is the ability of perinatal data collections to report maternal health indicators accurately. A Canadian validation study found that gestational hypertensive disorders were more accurately coded (sensitivity $88 \%$ ) than other variables, for example fetal/birth asphyxia (sensitivity 14.3\%) [34]. The study is further limited by lack of data regarding whether or not women had a hypertensive disorder during a previous pregnancy as risk factors for recurrence are likely to be different for those women whose pregnancy is affected for the first time. The use of measured, rather than self-reported, anthropometric variables is an advantage in the study particularly because of the importance of body mass and central adiposity in predicting hypertension in pregnancy.

\section{Conclusions}

Our study findings have enhanced our knowledge base, in general, about risk for hypertension in pregnancy. These data demonstrate high levels of obesity and poor metabolic health among Aboriginal and Torres Strait Islander women of child-bearing age in north Queensland that increase the risk for hypertensive disorders of pregnancy. Levels of physical activity were low. The study was undertaken in remote communities situated in isolated areas of Cape York and on Torres Strait Islands that frequently have limited resources for access to secure supplies of healthy foods and safe exercise environments. As well as improving food supply, consideration should be given to improving community infrastructures to include identified facilities (gymnasiums, walking paths and improved night lighting) which provide physical activity programs aimed at improving the health of young women prior to their first pregnancy and between pregnancies. Beneficial effects of better metabolic health of a woman of childbearing age may also have a strong positive effect on the health of her children across their lifespan.

\section{Competing interests}

The authors declare that they have no competing interests.

\section{Authors' contributions}

The contributions of each author in this work are SC performed the data analysis, interpreted the data and drafted the manuscript. RM conceived and implemented the baseline study and linked hospitalisations data. JL, AE and RM contributed to data analysis and critical revision of the manuscript. All authors read and approved the final manuscript.

\section{Acknowledgements}

This work was supported by a Postgraduate Scholarship (Award Reference No PP08 A 4066) from the National Heart Foundation of Australia, by NHMRC Project Grant 456402 and in part by NHMRC Project Grant 279402. $J$ is supported by an NHMRC Australia Fellowship. RM is supported by a NHMRC Practitioner Fellowship. The authors would like to thank the health staff in the participating communities, and Apunipima and the Torres Strait Islands and NPA Health Councils for their support for the project and the Brisbane Statistics Centre for matching and providing perinatal data. In particular, the authors extend their thanks to all the participants.

\section{Author details}

${ }^{1}$ School of Health Sciences, Division of Health Sciences, University of South Australia, City East Campus North Terrace (P5-21), GPO Box 2471, Adelaide, SA 5001, Australia. ${ }^{2}$ Discipline of Public Health, University of Adelaide, Adelaide, SA, Australia. ${ }^{3}$ School of Nursing and Midwifery, Division of Health Sciences, University of South Australia, Adelaide, SA, Australia. ${ }^{4}$ Sansom Institute, Division of Health Sciences, University of South Australia, Adelaide, SA, Australia.

Received: 16 August 2012 Accepted: 13 February 2013 Published: 15 February 2013

\section{References}

1. Lindheimer M, Taler S, Cunningham F: Hypertension in pregnancy. J Am Soc Hypertens 2010, 4:68-78.

2. Roberts J, Pearson G, Cutler J, Lindheimer M: Summary of the NHLBI working group on research on hypertension during pregnancy. Hypertension 2003, 41:437-445. 
3. Jim B, Sharma S, Kebede T, Acharya A: Hypertension in pregnancy. Cardiol Rev 2010, 18:178-179.

4. Roberts $\mathrm{CL}$, Algert CS, Morris JM, et al: Hypertensive disorders in pregnancy: a population-based study. Med J Aust 2005, 182:332-335

5. Jacobs D, Vreeburg S, Dekker G, et al: Risk factors for hypertension during pregnancy in South Australia. Aust NZJ Obstet Gynaecol 2003, 43:421-428.

6. De Costa C, Child A: Pregnancy outcomes in urban Aboriginal women. Med J Aust 1996, 164:523-526.

7. Leonard D, McDermott R, O'Dea K, et al: Measuring prevalence: obesity, diabetes and associated cardiovascular risk factors among Torres Strait Islander people. Aust N Z J Public Health 2002, 26:144-149.

8. Trewin D, Madden R: The health and welfare of Australia's Aboriginal and Torres Strait Islander peoples 2005. Canberra: Australian Bureau of Statistics and Australian Institute of Health and Welfare; 2005.

9. Sibai B, Dekker G, Kupferminc M: Pre-eclampsia. Lancet 2005, 365:785-799.

10. Gilbert W, Young A, Danielson B: Pregnancy outcomes in women with chronic hypertension: a population based study. J Reprod Med 2007, 52:1046-1051

11. Rodie V, Freeman D, Sattar N, Greer I: Pre-eclampsia and cardiovascular disease: metabolic syndrome of pregnancy? Atherosclerosis 2004, 175:189-202.

12. Roberts J, Bodnar L, Patrick T, Powers R: The role of obesity in preeclampsia. Pregnancy Hypertens 2011, 1:6-16.

13. Panaretto $K$, Lee $H$, Mitchell $M$, et al: Risk factors for preterm, low birth weight and small for gestational age birth in urban Aboriginal and Torres Strait Islander women in Townsville. Aust N Z J Public Health 2006 30:163-170.

14. Abenhaim $H$, Kinch $R$, Morin L, et al: Effect of prepregnancy body mass index categories on obstetrical and neonatal outcomes. Arch Gynecol Obstet 2007, 275:39-43

15. Samuels-Kalow M, Funai E, Buhimschi $C$, et al: Prepregnancy body mass index, hypertensive disorders of pregnancy, and long-term maternal mortality. Am J Obstet Gynecol 2007, 197:490-e491. 490.e496.

16. Roberts J, Bodnar L, Patrick T, Powers R: The role of obesity in preeclampsia. Pregnancy Hypertens 2011, 1:6-16.

17. O'Dea K: Preventable chronic disease among Indigenous Australians: the need for a comprehensive national approach. Heart Lung Circ 2005, 14:167-171.

18. McDermott R, Campbell S, Li M, McCulloch B: The health and nutrition of young Indigenous women in north Queensland - intergenerational implications of poor food quality, obesity, diabetes, tobacco smoking and alcohol use. Public Health Nutr 2009, 12:2143-2149.

19. Miller G, McDermott R, McCulloch B, et al: The Well Person's Health Check: a population screening program in Indigenous communities in north Queensland. Aust Health Rev 2002, 25:136-147.

20. Campbell S, Lynch J, Esterman A, McDermott R: Pre-pregnancy predictors of diabetes in pregnancy among Aboriginal and Torres Strait Islander women in North Queensland, Australia. Matern Child Health J 2011 doi:10.1007/s10995-011-0889-3.

21. Flenady V, King J, Charles A, et al: Perinatal Society of Australia and New Zealand (PSANZ) Clinical Practice Guidelines for Perinatal Mortality. 2nd edition. Canberra: PSANZ; 2009.

22. World Health Organisation: Obesity: preventing and managing the global epidemic. Geneva: Report of a WHO consultation; 2000.

23. McCulloch B, McDermott R, Miller G, et al: Self-reported diabetes and health behaviours in remote Indigenous communities in Northern Queensland, Australia. Diabetes Care 2003, 26:397-403.

24. Coyne KL, De Costa CM, Heazlewood RJ, Newman HC: Pregnancy characteristics of women giving birth to children with fetal alcohol syndrome in Far North Queensland. Aust N Z J Obstet Gynaecol 2008, 48:240-247.

25. Hatzis C, Bertsias G, Linardakis M, et al: Dietary and other lifestyle correlates of serum folate concentrations in a healthy adult population in Crete, Greece. Nutr J 2006. doi:10.1186/1475-2891-5-5.

26. Hoy W, McDonald S: Albuminuria: marker or target in Indigenous populations. Kidney Int 2004, 92:S25-S31.

27. Callaway L, Prins J, Chang A, McIntyre H: The prevalence and impact of overweight and obesity in an Australian obsteric population. Med J Aust 2006, 184:56-59.

28. Duckitt K, Harrington D: Risk factors for pre-eclampsia at antenatal booking: systematic review of controlled studies. BMJ 2005, 10.1136/ bmj.38380.674340.EO.
29. Shaw K, Gennat H, O'Rourke P, Del Mar C: Exercise for overweight or obesity. Cochrane Database Syst Rev 2006, (4). doi:10.1002/14651858. CD003817.pub3.

30. Mertens I, Van Gaal L: Overweight, obesity, and blood pressure: the effects of modest weight reduction. Obesity 2000, 8:270-278.

31. Neter J, Stam B, Kok F, et al: Influence of weight reduction on blood pressure. A meta-analysis of randomized controlled trials. Hypertension 2003, 42:878-884.

32. Rudra C, Williams $M$, Lee l, et al: Perceived exertion during prepregnancy physical activity and preeclampsia risk. Medicine and Science in Sports \& Exercise 2005, 37:1836-1840.

33. Zavorsky G, Longo L: Exercise guidelines in pregnancy: new perspectives. Sports Med 2011, 41:345-360

34. Joseph K, Fahey J: Validation of perinatal data in the Discharge Abstract Database of the Canadian Institute for Health Information. Chronic Dis Can 2009, 29:96-100.

\section{doi:10.1186/1471-2458-13-138}

Cite this article as: Campbell et al.: Pre-pregnancy predictors of hypertension in pregnancy among Aboriginal and Torres Strait Islander women in north Queensland, Australia;

a prospective cohort study. BMC Public Health 2013 13:138.

\section{Submit your next manuscript to BioMed Central and take full advantage of:}

- Convenient online submission

- Thorough peer review

- No space constraints or color figure charges

- Immediate publication on acceptance

- Inclusion in PubMed, CAS, Scopus and Google Scholar

- Research which is freely available for redistribution

Submit your manuscript at www.biomedcentral.com/submit
C) Biomed Central 